\title{
Importance of the embedding environment on the strain within small rings in siliceous materials
}

\author{
Stefan T. Bromley, Ibério de P. R. Moreira, and Francesc Illas \\ Departamento de Química Física \& Centre Especial de Recerca en Química Teórica, Universitat de Barcelona \\ \& Parc Científic de Barcelona, C/ Martí i Franquès 1, E-08028 Barcelona, Spain \\ Jacek C. Wojdeł \\ Ceramic Membrane Centre “The Pore,” Delft University of Technology, Julianalaan 136, 2628 BL, Delft, The Netherlands
}

(Received 23 January 2006; published 10 April 2006)

\begin{abstract}
The effect of the local environment on the energetic strain within small $(\mathrm{SiO})_{N}$ rings (with $\left.N=2,3\right)$ in silica materials is investigated via periodic model systems employing density functional calculations. Through comparison of the energies of various nonterminated systems containing small rings in strained and relatively unstrained environments, with alpha quartz, we demonstrate how small ring strain is affected by the nature of the embedding environment. We compare our findings with numerous previously reported calculations, often predicting significantly different small-ring strain energies, leading to a critical assessment of methods of calculating accurate localized ring energies. The results have relevance for estimates of the strain-induced response (e.g., chemical, photo, and radio) of small silica rings, and the propensity for them to form in bulk glasses, thin films, and nanoclusters.
\end{abstract}

DOI: 10.1103/PhysRevB.73.134202 PACS number(s): 61.43.-j, 68.35.Gy, 71.15.Mb, 91.60.Ed

\section{INTRODUCTION}

Theoretical and experimental studies have confirmed the existence of rings containing two $\mathrm{SiO}$ units (two-rings) on the surfaces of, and rings containing three $\mathrm{SiO}$ units (threerings) on and within reconstructed and dehydroxylated crystalline silicas, ${ }^{1-3}$ thin films. ${ }^{4,5}$ silica glasses ${ }^{3,6-8}$ and silica nanoparticles. ${ }^{5,9}$ Both ring types are strain-activated reactive centers, ${ }^{6}$ which are thought to play an important role in stress corrosion and mechanical hydrolytic weakening of silica. ${ }^{10}$ Small silica rings have also been put forward as likely electron traps which, under external stimuli from light or radiation, give rise to $E^{\prime}$-type defects. ${ }^{11,12}$ The presence of such defects deeply affects the quality of optical and electronic devices (e.g., optical fibres, transistor gate dielectrics), which rely on high-purity silica for their performance. In order to understand the formation and stability of small rings, and thus minimize their detrimental impact in technical applications, it is imperative that we have an accurate assessment of the inherent strain with these centers and how the type of siliceous environment in which they reside affects this strain.

Most estimates of small-ring strain have used calculations employing either terminated silica clusters or periodic silicas containing small rings as model strained systems (MS systems). The energy of a relatively unstrained system (U system) of a suitable corresponding type is then subtracted from that of the MS system in order to evaluate the excess smallring strain energy. In such calculations it is assumed that the strain energy of a small ring is very localized, and thus all the difference in energy between the MS and U systems comes from the small ring in question. In MS systems, where a small ring is embedded in an extended unstrained silica environment or is simply terminated by freely relaxed terminating groups, it seems quite clear that by employing the corresponding type of $U$ system the energies of the unstrained and/or relaxed parts of each system will cancel, leaving the energy difference of a small ring and unstrained silica.
The first reported estimates of small ring strain were based upon Hartree-Fock (HF) calculations of Born-Haber reaction cycles ${ }^{13-15}$ using small $\mathrm{OH}$-terminated cluster models. Essentially such methods can be classed as subtractive methods where the energy of the relaxed siliceous "reactants" is subtracted from the energy of the small-ringcontaining "products" to give an estimate of the energy required to form a small ring. One of the more reliable calculations of this type gives an estimated two-ring strain of $1.83 \mathrm{eV}$ and a three-ring strain of $0.81 \mathrm{eV} .{ }^{15}$ Other, more recent subtractive cluster based approaches using both HF 16 and density functional theory (DFT) (Ref. 17) do not rely on explicit reaction schemes but, nevertheless, as with the former type of calculation, assume that the terminating species (typically $\mathrm{OH}$ groups) employed in the $\mathrm{U}$ system and the MS system clusters possess the same energy in both systems. Using an elegant subtractive technique Uchino et al. ${ }^{16} \mathrm{em}$ ployed four different $\mathrm{Si}_{9} \mathrm{O}_{25} \mathrm{H}_{14}$ clusters to give Hartree-Fock (HF) calculated estimates of the strain energies of two-rings and three-rings of 1.85 and $0.26 \mathrm{eV}$, respectively. In another subtractive cluster calculation, using DFT and based directly on $\mathrm{OH}$-terminated small rings of sizes $(\mathrm{SiO})_{N} N=2-4,{ }^{17}$ two- and three-ring strain energies were estimated to be 1.42 and $0.24 \mathrm{eV}$. The two-ring angular strain energy quoted in Ref. 17 of $0.71 \mathrm{eV}$ is that of an $\mathrm{OH}$-terminated two-ring normalized to its two constitutive $\mathrm{O}-\mathrm{Si}(\mathrm{OH})_{2}$ units. The total energy per two-ring is thus twice this value, giving $1.42 \mathrm{eV}$ per two-ring. In the Discussion section below, we examine the choices of theory and/or MS system in some of these cluster subtractive calculations in order to assess their adequacy for refined measures of minimal small-ring strain energies.

One feature excluded from all cluster subtractive calculations is the provision for an extended nonterminated siliceous environment to better represent the embedding environment within which small rings actually exist in real 
<smiles>[O-][Si]([O-])([O-])O[Si]([O-])([O-])[O-]</smiles>

(a)
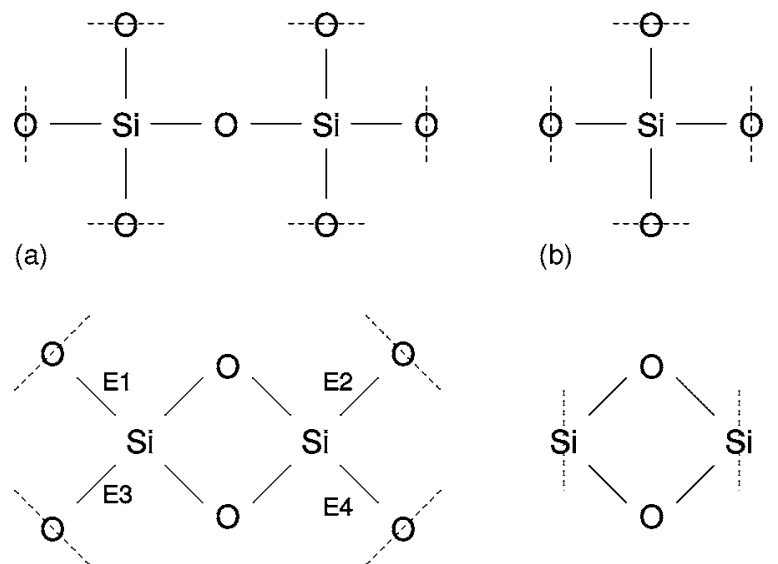

(c)

(d)

FIG. 1. Schematic representations of $\mathrm{Si}_{2} \mathrm{O}_{4}$ and $\mathrm{SiO}_{2}$ units when taken to be a part of a relaxed siliceous material (a) and (b) and when constituting a strained two-ring (c) and (d).

siliceous materials. This point has been particularly stressed by Hamman, ${ }^{8}$ who performed the first periodic DFT subtractive calculations to obtain two- and three-ring strain energies. This study is often regarded as providing benchmark strain energies for two- and three-rings embedded in siliceous materials. It is noted that these periodic subtractive calculations yielded relatively low small-ring strain energies compared to subtractive cluster calculations, indicating the importance of including an embedding material environment in such studies.

For subtractive cluster calculations the U and MS system energies are just the total respective cluster energies, whereas for periodic systems energies are always necessarily per some repeat unit of the material. This simple observation becomes important when one considers subtractive calculations using MS systems with varying densities of small rings. As an illustrative example, we take two extreme cases of periodic subtractive calculations of two-ring strain from the literature: (i) a MS system consisting of a large unit cell containing over 50 atoms and only one two-ring, ${ }^{3}$ and (ii) an MS system with a unit cell consisting of only two-rings. ${ }^{18}$ We show that employing the same units $\left(\mathrm{eV} / \mathrm{Si}_{2} \mathrm{O}_{4}\right)$ for the energy per two-ring in both cases gives quite different values of the resulting two-ring strain energy. In fact, the subtractive procedure in each study should indeed give quite similar energies per two-ring but only when taking account of the respective density of two-rings in each case.

In Figs. 1(c) and 1(d) representations of $\mathrm{SiO}_{2}$ and $\mathrm{Si}_{2} \mathrm{O}_{4}$ units are schematically shown, each corresponding to a way of partitioning a two-ring from its environment. For the $\mathrm{SiO}_{2}$ unit we have simply the four $\mathrm{Si}-\mathrm{O}$ bonds that constitute the internal energy of the ring. In the case of the $\mathrm{Si}_{2} \mathrm{O}_{4}$ unit we have in addition four further $\mathrm{Si}-\mathrm{O}$ bonds. In the first study ${ }^{3}$ the MS system is taken to be a large supercell slab of cristobalite containing one reconstructed surface with a single two-ring and another hydroxylated suface. Subtracted from the total energy of this system is (i) half the energy of a fully hydroxylated supercell slab of the same size, together with (ii) the energy of the number of $\mathrm{SiO}_{2}$ units in the remaining half-slab taken to have the energy per $\mathrm{SiO}_{2}$ of relaxed bulk

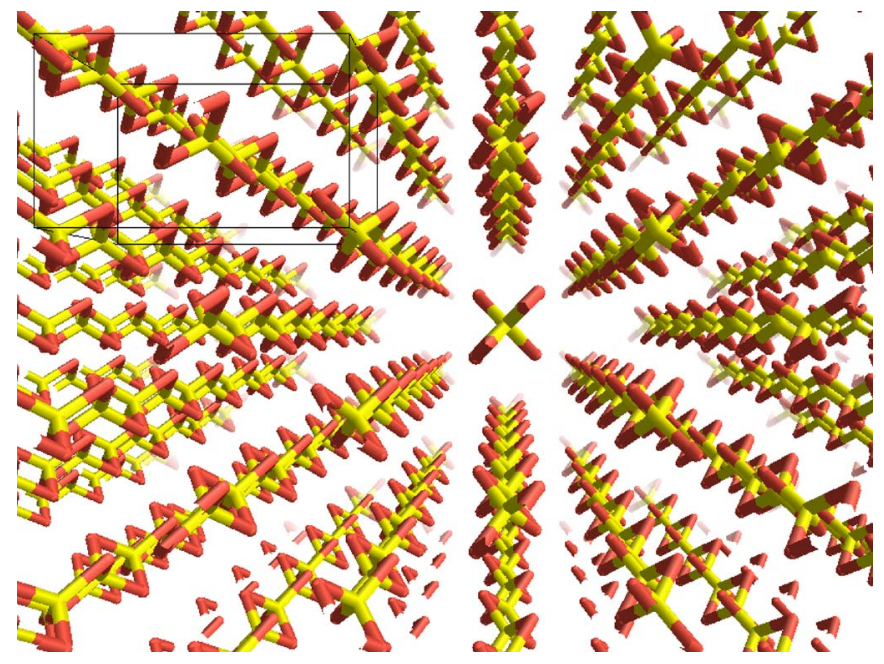

FIG. 2. (Color online) Perspective view of the two-ring-chainbased silica- $w$ material looking down the $c$ axis.

cristobalite. If we use the units of $\mathrm{eV} / \mathrm{Si}_{2} \mathrm{O}_{4}$ to express the final energy difference, we will figuratively have the energy of the unit expressed in Fig. 1(c). As the two-ring in the MS system is linked to its surroundings via four $\mathrm{Si}-\mathrm{O}$ bonds with a similar energy to those in normal cristobalite [i.e., the $\mathrm{Si}-\mathrm{O}$ bonds $E 1-E 4$ in Fig. 1(c) are energetically similar to those of Fig. 1(a)], the contribution of these "extra" bonds to the energy difference will largely cancel, essentially leaving the energy of only the four Si-O internal bonds of the tworing. Such a procedure is found to yield an energy of $1.38 \mathrm{eV}$ per two-ring. ${ }^{3}$ In fact, for such a MS system, as we only have one two-ring per unit cell and if we assume that the Si-O bond energies external to the two-ring are canceled in the subtraction procedure, any energy unit with respect to at least four $\mathrm{Si}-\mathrm{O}$ bonds, up to the size of the unit cell itself, is adequate.

In systems where the small rings in the MS system are in an environment possessing considerable strain, the cancellation of the extra $\mathrm{Si}-\mathrm{O}$ bond energy [i.e. of $E 1-E 4$ in Fig. $1(c)]$ is however not complete. In such cases the use of the $\mathrm{eV} / \mathrm{Si}_{2} \mathrm{O}_{4}$ unit leads to a significant additional term to the energy difference between MS and $\mathrm{U}$ systems, and thus a correspondingly increased measure of small-ring strain. This effect of the immediate embedding environment on $\mathrm{calcu}$ lated small-ring strain is clearly highlighted in the type of MS system employed in Ref. 18. Here, a material consisting solely of discrete chains of connected two-rings ( silica- $\left.w^{9}\right)$ is employed as the MS system and the energy compared with that of alpha-quartz per $\mathrm{Si}_{2} \mathrm{O}_{4}$. In silica- $w$, which can ideally be considered as a concatenation of the $\mathrm{SiO}_{2}$ units shown in Fig. 1(d), the number of two-rings corresponds to the number of $\mathrm{SiO}_{2}$ units (see Fig. 2).

Thus, the energy per the four Si-O bonds in every tworing in silica- $w$ is simply the energy per $\mathrm{SiO}_{2}$ unit. Calculating the energy subtraction using units of $\mathrm{eV} / \mathrm{Si}_{2} \mathrm{O}_{4}$ means that we also take into account the extra strained $\mathrm{Si}-\mathrm{O}$ bonds [i.e. $E 1-E 4$ in Fig. 1(c)] which, due to the structure of silica$w$, are also within two-rings. The energetic difference of such bonds with the corresponding unstrained bonds in the alpha- 
quartz $\mathrm{U}$ system is significant (actually the energy of another two-ring), leading to a calculated two-ring strain energy of $2.46 \mathrm{eV} / \mathrm{Si}_{2} \mathrm{O}_{4}$. The value quoted in Ref. 18 in Table $\mathrm{V}$ is too small by a factor of two due to the use of $\mathrm{eV} / \mathrm{Si}_{2} \mathrm{O}_{4}$ units rather than $\mathrm{eV} / \mathrm{SiO}_{2} \cdot{ }^{20}$ Otherwise, in the paper the calculated energy per two-ring in silica- $w$ is correctly stated as being $1.23 \mathrm{eV}$. The value of $1.23 \mathrm{eV} / \mathrm{SiO}_{2}$ (i.e., $1.23 \mathrm{eV}$ per tworing in silica- $w$ ) is comparable to that found for two-ring on the reconstructed cristobalite surface. ${ }^{3}$ In each case, although the units are different it is the final energy per two-ring which is the important value.

The value of $1.23 \mathrm{eV}$ per two-ring from the subtractive silica- $w$ calculation ${ }^{18}$ was found to be the lowest estimate of two-ring strain reported in the literature, and has become an important benchmark value. Similar periodic subtractive calculations on three-rings in the same study ${ }^{16}$ also predict relatively small three-ring strain energies. In this work we assess the use of silica- $w$ as a two-ring MS system employing a range of different methodologies and levels of theory using periodic subtractive calculations. Further extending this idea, we test a number of MS systems, which have been deliberately designed to vary the degree of environmental strain placed on the embedded small rings (both two-rings and three-rings). We investigate how all these choices affect the resulting small-ring strain energies obtained.

\section{METHODOLOGY}

In order to assess the effect of the choice of calculation methodology on calculated small-ring strain, we performed a series of periodic DF and HF calculations on the silica- $w$ MS system and the U system (alpha quartz) with three methodologically different codes: VASP, ${ }^{21}$ CRYSTAL, ${ }^{22}$ and DACAPO, ${ }^{23}$ each with its particular features with respect to the description of the core electrons or in the type of basis set employed. The silica- $w$ material is made solely of discrete infinite chains of connected two-rings and is essentially onedimensional structurally (see Fig. 2). Interactions between the chains have been reported as being small enough to be negligible and to be an nonessential contribution to the calculation of the strain energy and geometry of a two-ring. ${ }^{24,18}$ In order to explicitly test this we also calculated the energies of both isolated two-ring chains and the interacting chain system of silica- $w$, finding, as we describe below, that interactions between two-ring-containing systems are in fact surprisingly important. For all other calculations reported for other two-ring- and three-ring-containing MS systems the VASP code was used exclusively.

For all VASP-calculated energies and structures, periodic DF calculations using the projected augmented wave ${ }^{25}$ method for core states and a plane-wave basis set were employed with the PW91 functional ${ }^{26}$ and suitable $k$-point meshes generated via the Monkhorst-Pack scheme. ${ }^{27}$ All calculations allowed for both internal atomic positions and cell dimensions to vary freely in order to obtain the lowest energy structures. A relatively large energy cutoff of $800 \mathrm{eV}$ was employed to minimize the spurious effects of basis set variability with changing cell size, and all final energies were checked by subsequent reoptimizations with a reset cutoff and finally via high-level single-point calculations.

Plane-wave pseudopotential (PW-PP) calculations were done using DACAPO software accessed through the CamposASE environment. ${ }^{22}$ As in the case of VASP calculations, we used the PW91 functional, ${ }^{25}$ with Monkhorst-Pack meshes of $k$ points. ${ }^{26}$ For all PW-PP calculations we used Vanderbilt ultrasoft pseudopotentials (USPPs). ${ }^{28}$ The silicon USPP was used as included in the 7.3.4 version of the USPP generation package, while the oxygen USPP had to be generated. The original USPP package does not contain oxygen USPP generated with the PW91 functional, so we used another USPP generated with the gradient-corrected functional as a template. As the transferability test included with the USPP returned essentially the same results as for the original one, no further testing of the oxygen USPP was done. In most of the calculations using DACAPO, we allowed ions to fully relax within fixed unit cell and used a $900 \mathrm{eV}$ kinetic energy cutoff. Only in some instances, the structural relaxation was done at $400 \mathrm{eV}$ cutoff, followed by a $900 \mathrm{eV}$ single-point energy calculation. The latter procedure has been tested to yield energies within $1 \mathrm{meV}$ difference from full optimization at $900 \mathrm{eV}$ cutoff. Unit cell optimizations using DACAPO code have been done only for the silica- $w$ unit cell, using the stress tensor and conjugated gradients optimization procedure. Calculations of alpha quartz were done at the experimental unit cell geometry.

Periodic all-electron calculations have been performed by using the periodic HF method, ${ }^{29,30}$ and several DFT approximations (LDA, GGA, and B3-LYP), ${ }^{31}$ as implemented in the CRYSTAL03 code. $^{22}$ The LDA Hamiltonian is based on approximate local functionals for both the exchange and the correlation effects. The S-VWN parametrization was used, according to Slater's exchange ${ }^{32}$ and Vosko-Wilk-Nusair's correlation $^{33}$ potentials. The gradient corrected approximation (GGA) used is based on the Perdew and Wang exchange-correlation functional. ${ }^{34}$ Finally, the B3-LYP functional is a combination of Becke's ${ }^{35}$ and Hartree-Fock potentials for the exchange effect and uses the gradient-corrected correlation functional of Lee, Yang, and Parr. ${ }^{36}$ All-electron localized basis sets used to represent the atomic orbitals of $\mathrm{Si}$ and $\mathrm{O}$ have been reported in Ref. 37 with slightly different exponents for outermost $s p$ and $d$ functions $\mathrm{O}: a(p)=0.28$, $a(d)=0.8 ; \mathrm{Si}: a(p)=0.21, a(d)=0.45$. The same basis set was used to extract the relative stability of silica- $w$ with respect to alpha quartz. The level of numerical approximation in evaluating the Coulomb and exchange series appearing in the self-consistent fields (SCF) equations for periodic systems is controlled by the ITOL, which were set to 7777 and 14 strict values. The reciprocal space was sampled according to a regular sublattice defined by seven points in the irreducible Brillouin zone for alpha quartz. For silica- $w$ four points have been used in the irreducible Brillouin zone.

\section{RESULTS}

In the following subsections we report our finding for small-ring strain employing various computational methodologies, levels of theory, and types of MS system. 
TABLE I. Summary of periodic calculations of silica- $w$ performed with CRYSTAL (Ref. 21) VASP (Ref. 21), custom plane-wave code (Refs. 18 and 38), DACAPO (Ref. 23). For calculation with VASP and DACAPO a $3 \times 3 \times 4$ grid of $k$ points was employed. The calculated $c$ lattice parameters of silica- $w$ and the isolated two-ring chains are compared in parentheses to the experimental value of $4.72 \AA$ for silica- $w$ (Ref. 19). For further comparison, we also give the ranges of bond lengths and angles for alpha quartz [optimized employing VASP (Ref. 21 ) with a $2 \times 2 \times 2$ supercell, the PW91 functional, and a $4 \times 4 \times 4$ grid of $k$ points]: $\mathrm{Si}-\mathrm{O}$ bond lengths (1.627-1.632), O-Si-O angles (108.7-110.5), Si-O-Si angles (144.1).

\begin{tabular}{|c|c|c|c|c|c|c|}
\hline Theory & $\begin{array}{c}c \text { lattice } \\
\text { parameter of } \\
\text { silica-W }(\AA)\end{array}$ & $\begin{array}{c}c \text { lattice } \\
\text { parameter of an } \\
\text { isolated two- } \\
\text { ring chain }(\AA)\end{array}$ & $\begin{array}{c}\text { Energy per } \\
\text { two-ring } \\
\text { with respect to quartz } \\
\left(\mathrm{eV} / \mathrm{SiO}_{2}\right)\end{array}$ & $\begin{array}{l}\text { Si-O bond } \\
\text { length }(\AA)\end{array}$ & $\begin{array}{l}\mathrm{O}-\mathrm{Si}-\mathrm{O} \\
\text { angle }\left(^{\circ}\right)\end{array}$ & $\begin{array}{l}\text { Si-O-Si } \\
\text { angle }\left(^{\circ}\right)\end{array}$ \\
\hline $\mathrm{HF}^{\mathrm{a}}$ & & $4.695(-0.025)$ & $-/ 1.73$ & $-/ 1.644$ & $-/ 91.0$ & $-/ 89.0$ \\
\hline $\mathrm{HF}^{\mathrm{a}}$ (Ref. 24) & & $4.68(-0.04)$ & $-/ 2.12$ & $-/ 1.643$ & $-/ 90.9$ & $-/ 89.1$ \\
\hline $\mathrm{LDA}^{\mathrm{a}}$ & & $4.661(-0.059)$ & $-/ 1.58$ & $-/ 1.664$ & $-/ 91.1$ & $-/ 88.9$ \\
\hline B3LYPa & & $4.716(-0.004)$ & $-/ 1.48$ & $-/ 1.699$ & $-/ 90.1$ & $-/ 89.9$ \\
\hline PW91 ${ }^{\mathrm{a}}$ & & $4.712(-0.008)$ & $-/ 1.41$ & $-/ 1.681$ & $-/ 91.1$ & $-/ 88.9$ \\
\hline PW91 ${ }^{\mathrm{b}}$ & $4.731(+0.011)$ & $4.707(-0.013)$ & $1.26 / 1.37$ & $1.673 / 1.672$ & $90.0 / 90.6$ & $90.0 / 89.4$ \\
\hline PW91c (Ref. 18) & $4.757(+0.037)$ & & $1.23 /-$ & $1.678 /-$ & $89.7 /-$ & $90.3 /-$ \\
\hline PW91 ${ }^{\mathrm{d}}$ & $4.701(-0.019)$ & & $1.19 /-$ & $1.674 /-$ & $90.5 /-$ & $89.5 /-$ \\
\hline
\end{tabular}

${ }^{\mathrm{a}}$ CRYSTAL (Ref. 21).

b VASP (Ref. 21).

${ }^{\mathrm{c}}$ Custom plane-wave code (Refs. 18 and 38).

${ }^{\mathrm{d}}$ DACAPO (Ref. 23).

\section{Two-ring chains}

In Table I we summarize our calculations of two-ring strain in silica- $w$ (see Fig. 2) as judged via the energy per $\mathrm{SiO}_{2}$ unit with respect to that of alpha quartz using periodic calculations employing different levels of theory and computational setup. Also included for comparison are two reported values of two-ring strain in silica- $w$ from the literature, each calculated using exactly the same methodology as employed herein.

Table I contains the results of a series of calculations performed on both isolated infinite two-ring chains and on the packed two-ring chain system of silica- $w$. Where necessary the isolated two-ring chain was taken to be a periodic system of chains separated from each other by $12 \AA$ in the $a-b$ plane. Taking first the three calculations performed on the silica- $w$ system with the PW91 gradient corrected functional (bottom three entries of Table I), we can see that the energy per two-ring is consistently predicted to be $\sim 1.225 \mathrm{eV}$ $( \pm 0.035)$, forming the lowest range of calculated two-ring energies. In comparison, the two PW91 calculations of tworing strain in an isolated two-ring chain give $\sim 1.39 \mathrm{eV}$ $( \pm 0.020)$, which is a significant $(\sim 13.5 \%)$ increase over the value in silica- $w$. The most direct comparison can be made between respective calculations done with the same computational setup and functional (VASP/PW91), where we can directly see an increase in energy per $\mathrm{SiO}_{2}$ unit from 1.26 to $1.37 \mathrm{eV}(+8.7 \%)$. The lower relative energy calculated per two-ring in silica- $w$ is due to the interchain interactions and as such should not be ascribed to the four bonds within the two-rings but rather at the interaction between two-rings in different chains. The energy ascribed to the interactions between two-ring chains $\left(\sim 0.11 \mathrm{eV} / \mathrm{SiO}_{2}\right)$ would be surprisingly high for a van de Waals-type interaction in an inorganic material, as has been suggested to be the cause of the interchain attraction in Refs. 18 and 24, but is of a typical strength for a hydrogen bonded system. Although it is known that the application of DFT methods to systems with weak dispersive interactions is problematic, for electrostatically dominated hydrogen bonded systems such as the water dimer most pure GGA and hybrid functionals provide a good estimate (within 10\%) of the interaction energy from experiment and high-level $a b$ initio calculations. ${ }^{39}$ Based upon our GGA-calculated interaction strength we suggest that the interchain interaction is mainly of electrostatic origin, perhaps resulting from the dipolar nature of the constituent semiionic Si-O bonds in the chains. We note also that the interchain interaction is not totally passive with respect to the structure of the chains, with a small expansion of the $c$ parameter by $0.024 \AA$ occurring when isolated chains are brought together to form silica- $w$. This structural difference between isolated chains and interacting chain appears to arise almost solely because of small angular distortions induced in the two-rings rather than changes in the lengths of the constituent $\mathrm{Si}-\mathrm{O}$ bonds. Due to the sensitivity of the energy and structure of the two-ring chains upon their mutual interaction, we prefer to concentrate on the noninteracting isolated two-ring chain as a MS system in order to evaluate two-ring strain energies.

For the remaining isolated chain calculations the largest two-ring energies are given by the HF and LDA calculations. Although the predicted $c$ parameter for the isolated chain calculations is likely to be slightly contracted with respect to that calculated for the condensed material, comparison with the experimental $c$ lattice parameter gives an indication of the quality of the isolated chain results. The predicted $c$ lat- 
tice parameter closest to the experimental value of $4.72 \AA$ (Ref. 20) is given by the localized basis set CRYSTAL calculations using the B3LYP and PW91 functionals and also by the plane-wave VASP PW91 calculation, all giving only very slightly contracted values of $c$ (lower by $0.004-0.013$ ) with respect to the experimental value. The same set of three calculations also predicts very similar two-ring strain energies: $\sim 1.425 \mathrm{eV}( \pm 0.055)$. In contrast, the HF and LDA calculations listed in Table I all give relatively high and inconsistent two-ring energies and significantly contracted geometries compared to experiment.

With respect to the agreement with the experimentally determined geometry, the internal consistency of predicted magnitude of two-ring strain and the known improvements of GGA and hybrid functionals over HF and LDA approaches, the CRYSTAL calculations using B3LYP and PW91 and the VASP calculations using the PW91 functional appear to give a relatively better description of the two-ring chain MS system. In the remainder of the paper all reported calculations are performed using the VASP code with the PW91 functional.

\section{Two-ring strain energies in alternative MS systems}

In comparing the energies $\left(\mathrm{eV} / \mathrm{SiO}_{2}\right)$ of an isolated tworing chain and alpha quartz we cleanly calculate the average energy difference between a two-ring and a $\mathrm{SiO}_{2}$ unit in the most unstrained (lowest energy) polymorph known. Although in this case we can be sure that we have only tworings in our MS system (i.e., and no terminating groups or other types of Si-O bonds), we cannot assess how the energy of the four Si-O bonds within a two-ring are affected by being physically embedded within in an infinite chain of other two-rings. In order to gauge the effect of the directly bonded embedding environment of a two-ring upon the internal strain energy of systems we consider a range of MS systems in which the size of the rings immediately embedding the two-rings are systematically varied: $\mathrm{Si}_{N} \mathrm{O}_{N} N=2$ (silica-w) , 3, 4, 6. In addition, to further induce environmental strain on the two-rings in such systems, we consider the effect of applying topological distortions to the end members of this range.

For straight two-ring chains one of the simplest topological distortions one can apply to form a new periodic MS system is to bend regular segments of the chains upon themselves to form an array of closed rings of two-rings (see Fig. 3). Such systems were first considered in the context of investigating the energetic balance between internal strain and defect termination in finite two-ring chains, showing that fully coordinated rings were energetically favored over terminated finite chains for relatively small chain lengths. ${ }^{40}$

A periodic two-ring chain MS system is ideally infinite and thus devoid of energetically disfavored terminating defects. The internal strain is thus not compensated in the formation of an array of two-ring rings and the energy per tworing increases significantly $(+0.19 \mathrm{eV}$ per two-ring for an array of $\mathrm{Si}_{24} \mathrm{O}_{48}$ rings—-see Table II).

In order to increase the ring size of the immediate embedding environment of a two-ring, we must employ MS sys-

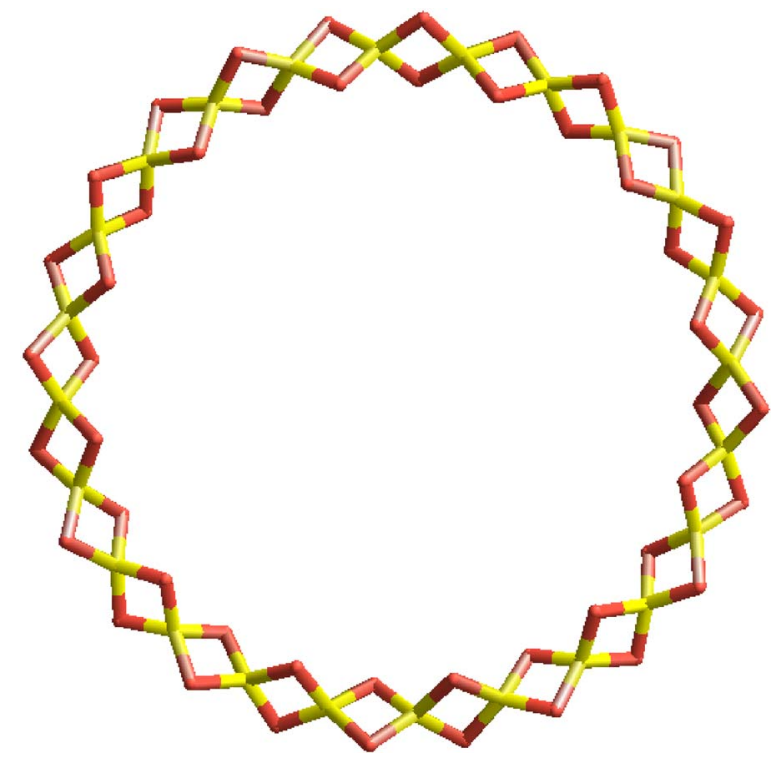

FIG. 3. (Color online) A silica two-ring ring $\left(\mathrm{Si}_{24} \mathrm{O}_{48}\right)$.

tems with richer topologies than periodic systems based upon simple one-dimensional chains and rings to accommodate the introduction of extra $\mathrm{Si}$ "vertices." Extending our repeat unit to two dimensions can be achieved by forming layered sheetlike materials consisting of a two-ring per every other $\mathrm{SiO}_{2}$ unit. Examples of four sheetlike materials are shown in Figs. 4(a)-4(d) with two-rings embedded by rings of size $\mathrm{Si}_{N} \mathrm{O}_{N} N=3,4$, and 6, respectively (referred to as $\mathrm{N}$-2-sheets hereafter). For the 6-2 embedding, two possible materials were assessed [see Figs. 4(c) and 4(d)] with the latter material (6-2-sheet-v2) found to have a slightly lower energy than the former (6-2-sheet_v1). Henceforth, "6-2sheet" refers to the lower energy 6-2-sheet_v2 material. In order to assess the relative energy per two-ring with respect to alpha quartz in such systems, one must remember that only half of the $\mathrm{SiO}_{2}$ units in the MS system are within two-rings. One way to do this is to calculate the energy per $\mathrm{SiO}_{2}$ with respect to alpha quartz and then weigh this value with respect to the proportion of $\mathrm{SiO}_{2}$ units per two-ring. Although this procedure always gives the energy per tworing, only in the ideal case of a system with two-rings in a perfectly unstrained environment (i.e., with $\mathrm{Si}-\mathrm{O}$ bond energies of alpha quartz) does this procedure yield exactly the energy of a two-ring in the system. Generally, however, the environmental embedding energy per $\mathrm{SiO}_{2}$ in a MS system will not be perfectly canceled by the energy per $\mathrm{SiO}_{2}$ in alpha quartz giving rise to an "excess" energy. In fact, when the proportion of "normal" $\mathrm{SiO}_{2}$ bonds to two-rings is 2 , as for the sheet materials, this procedure effectively calculates the energy per $\mathrm{Si}_{2} \mathrm{O}_{4}$, which, as we saw above for the case of silica- $w$, introduces energy extra to that only of the tworings. Although it is difficult to accurately assign localized energetic contributions in a periodic system, intuitively we would expect that the energy per $\mathrm{Si}_{2} \mathrm{O}_{4}$ tends to the limit of that of an isolated two-ring as we progressively decrease the strain on its embedding environment towards that of alpha quartz.

In Fig. 5 we show the energy $\left(\mathrm{eV} / \mathrm{Si}_{2} \mathrm{O}_{4}\right)$ of a series of materials with the size of the rings immediately embedding 
TABLE II. Summary of periodic DFT calculations of different structures with high concentrations of two-rings using the vASP code and the PW91 functional. Isolated two-ring-containing structures were calculated using sufficient separating space around them to avoid significant mutual interactions. The nanotubes were calculated using $1 \times 5 \times 1$ Monkhorst-Pack line of $k$ points along the tube direction; all other materials were calculated using $4 \times 4 \times 4$ Monkhorst-Pack grid of $k$ points. For the MS systems in which the two-rings have more than one value for a specific parameter and/or two-rings with more than one geometry exists, the $\mathrm{min} / \mathrm{max}$ range of geometric parameters is given.

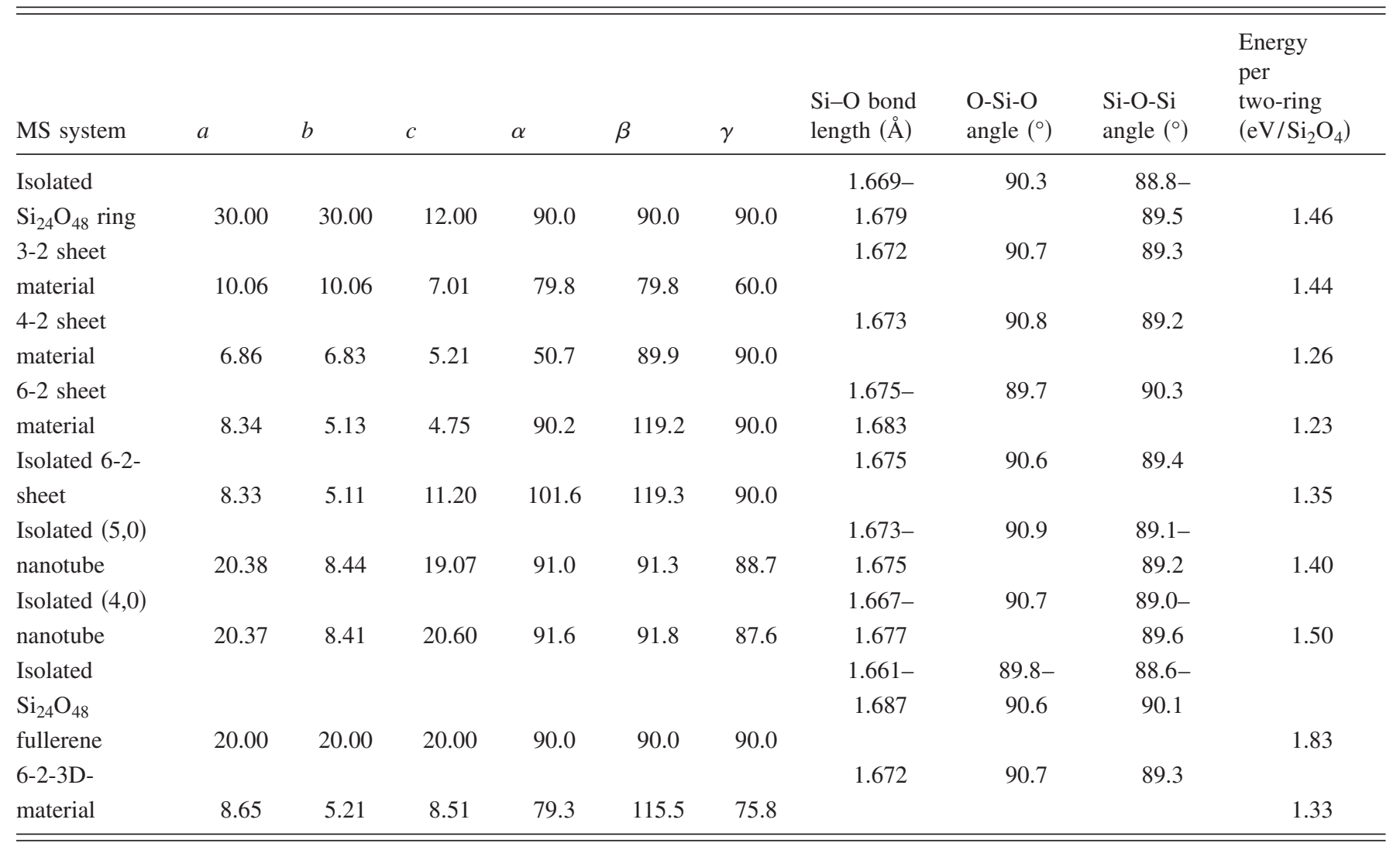

the two-rings increasing from $\mathrm{Si}_{2} \mathrm{O}_{2}$ to $\mathrm{Si}_{6} \mathrm{O}_{6}$ (silica-w, 3-2sheet, 4-2-sheet, 6-2-sheet_v2). As expected, the energy per $\mathrm{Si}_{2} \mathrm{O}_{4}$ unit tends towards a limiting value as the strain in the embedding bonding environment decreases with increasing $N$. Comparing the energy two-ring in the 6-2-sheet material $\left(1.23 \mathrm{eV} / \mathrm{Si}_{2} \mathrm{O}_{4}\right)$ with the energy per two-ring in silica-w $\left(1.26 \mathrm{eV} / \mathrm{SiO}_{2}\right)$, we see that the two-ring energy is slightly lower in the sheet material, indicating a small increase in the calculated two-ring strain energy due to a bonding environment consisting of strained rings rather than larger unstrained rings. In each case, however, the unit cell of each material was fully relaxed, allowing for energy-lowering intersheet/ interchain interactions. For a fairer comparison of two-ring strain energies in noninteracting two-ring chains and 6-2 sheets we also performed calculations of an isolated 6-2sheet, giving an energy per two-ring of $1.35 \mathrm{eV}$, compared to $1.37 \mathrm{eV}$ in an isolated two-ring chain. In both cases the MS system energy increases with respect to the corresponding interacting system, moreover, by a greater proportion than the change induced by varying the immediate ring size of the bonding environment. By reducing the influence of both bonding and nonbonding environments through an appropriate choice of MS system, we have refined our estimate of two-ring strain.

It is worth noting that the 6-2-sheet MS system can also be regarded as a structural analog to a graphene sheet by simply following the schematic formal analogy presented in Fig. 6. As for carbon-based graphene sheets, which can be thought of as providing the structural basis for carbon nanotubes and fullerenes, we can form silica-based analogous structures from the rolling-up 6-2-sheets. Using the carbon nanotube vector notation, we show in Figs. 7(a) and 7(b) $(4,0)$ and $(5,0) 6$ 6-2-sheet-based nanotubes, respectively.

For isolated silica nanotubes we see from Table II that the energy per $\mathrm{Si}_{2} \mathrm{O}_{4}$ unit is slightly increased $[+0.17(0,4)$-tube, $+0.27(0,5)$-tube $]$ with respect to the pure graphenelike isolated planar 6-2-sheet. We note that, although we use a structural analogy with experimentally realized carbon nanotubes, our silica nanotubes are quite unlike those currently formed by experimental means, ${ }^{41}$ which are typically amorphous and possess thicker walls. Our well-defined silica nanostructures are perhaps targets for future experiments, but for present purposes make for useful model systems for studying strain. Taking the analogy with carbon nanostructures further, we can also consider closed fullerenelike silica cages such as a $\mathrm{C}_{24}$ (Ref. 42) analog presented in Fig. 8 (possessing fourrings and six-rings as an immediate embedding environment to the two-rings). Such cagelike topologies significantly increase the strain energy per $\mathrm{Si}_{2} \mathrm{O}_{4}$ unit, with the calculated energy of the isolated $\mathrm{Si}_{24} \mathrm{O}_{48}$ fullerene found to be $1.83 \mathrm{eV} / \mathrm{Si}_{2} \mathrm{O}_{4}$ above alpha quartz. We have previously studied this and other types of silica cage in another study using the B3LYP functional and a localized $6-31 \mathrm{G}^{*}$ basis 


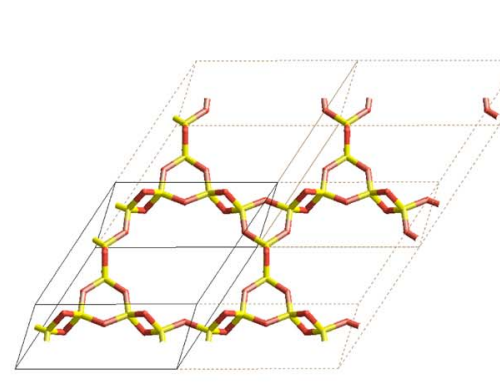

(a)

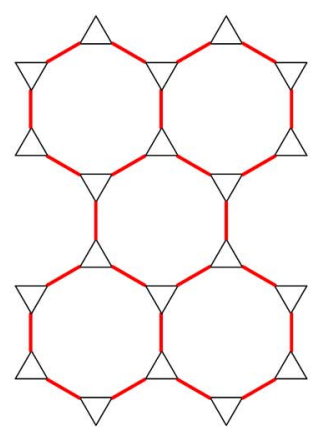

(b)
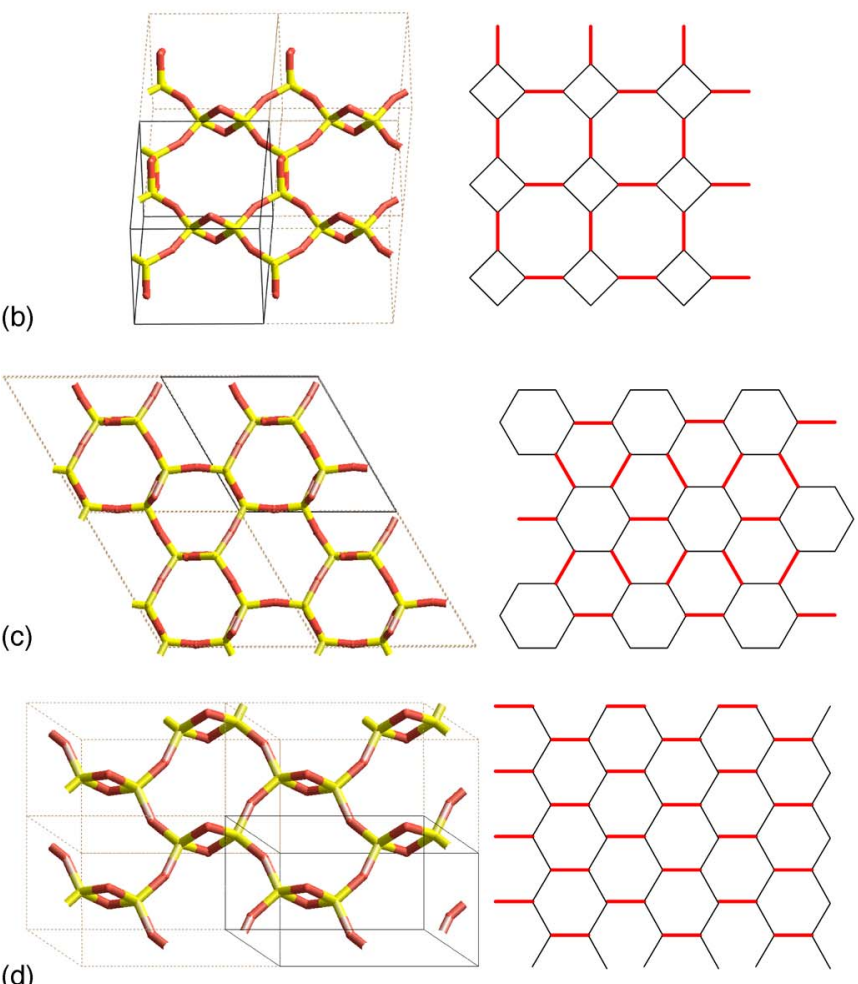

(d)

FIG. 4. (Color online) Sheetlike MS systems containing tworings embedded by three-rings [3-2-sheet-(a), four-rings (4-2sheet-(b), six-rings (6-2-sheet_v1-(c), and 6-2-sheet_v2-(d)]. The respective two-ring (solid bars) embedding is schematically represented on the right. The gray box in each figure, and in all subsequent figures, denotes the unit cell of the material.

set, which also yielded a very similar energy excess estimate for the $\mathrm{Si}_{24} \mathrm{O}_{48}$ fullerene with respect to quartz $\left(\sim 1.89 \mathrm{eV} / \mathrm{Si}_{2} \mathrm{O}_{4}\right)$, but which also noted its relative energetic stability with respect to other clusters of a similar size. $^{43}$

Although isolated 6-2-sheets provide a MS system with apparently low environmental bonding strain due to the large embedding six-rings, there still remains the question as to whether there may exists residual strain in the 6-ring due to the topological constraint of being in a two-dimensional sheet. In an attempt to relax this possible contribution to the environmental strain of the two-rings, we have further designed a three-dimensional two-ring-containing MS system with six-rings as immediate embedding rings (6-2-3Dmaterial: see Fig. 9). This material can be thought of as being formed from the concatenation of $\mathrm{Si}_{2} \mathrm{O}_{4}$ units as displayed in

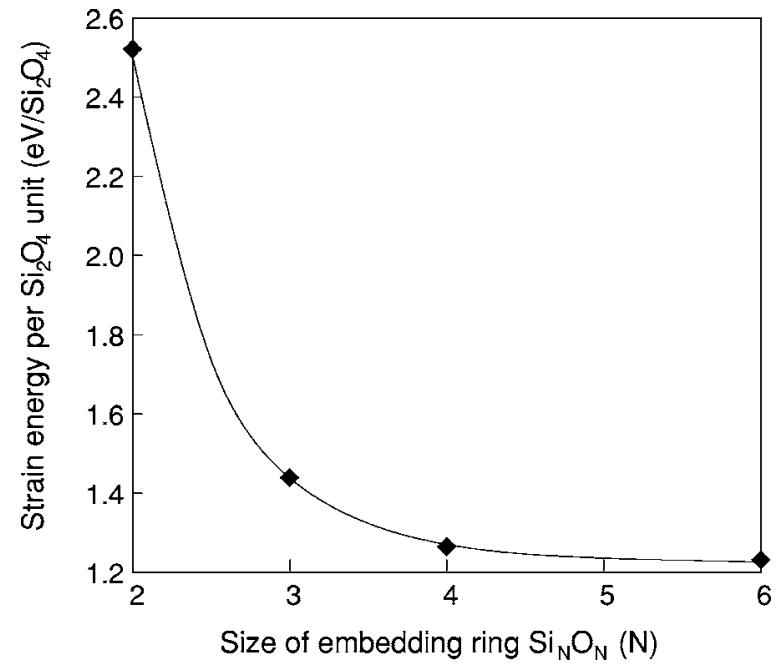

FIG. 5. The calculated energy per $\mathrm{Si}_{2} \mathrm{O}_{4}$ unit in a series of materials containing two-rings directly embedded by $\mathrm{Si}_{N} \mathrm{O}_{N}$ rings of varying size ( $N=2$ corresponds to silica- $w$ and $N=6$ to the 6-2-sheet material). The line is a guide to the eye.

Fig. 1(c), where for every $\mathrm{Si}_{2} \mathrm{O}_{4}$ unit four others join to it in mutally opposing directions, forming a natural tetrahedral building block. The energy of the 6-2-3D material formed in this way per $\mathrm{Si}_{2} \mathrm{O}_{4}$ is found to be $1.33 \mathrm{eV}$, even slightly lower than the isolated 6-2-sheet, perhaps indicating a small excess energetic contribution to the calculated two-ring strain energy in a more planar two-dimensional topology.

\section{Three-rings}

For assessing small-ring strain using periodic subtractive calculations, we have seen above that the MS system yielding the lowest ring strain is a three-dimensional material in which there is miminal ring-ring interation and embedding provided by large rings. These criteria are satisfied for threerings by a MS system proposed by Hamann, ${ }^{18}$ which contains three-rings in a three-dimensional network spaced by seven-rings [see Fig. 10(a)]. Our calculated energy of this material per three-ring $\left(0.21 \mathrm{eV} / \mathrm{Si}_{3} \mathrm{O}_{6}\right)$ is even slightly lower than that reported by Hamann $\left[0.25 \mathrm{eV} / \mathrm{Si}_{3} \mathrm{O}_{6}\right.$ (Ref. 18)] using a very similar methodology which may be ascribed to small differences in calculation methodologies. We

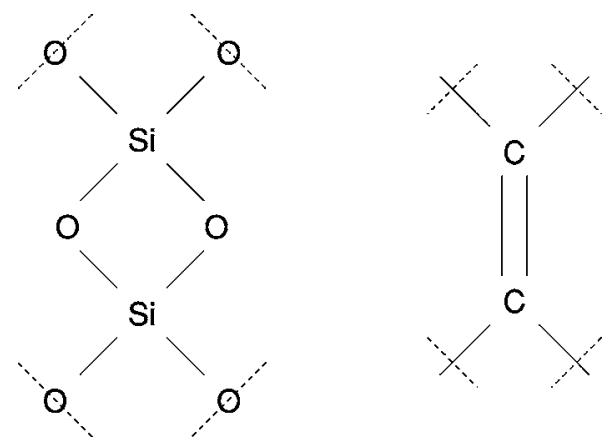

FIG. 6. Schematic representation of the formal bonding analogy between two-rings and carbon double bonds. 


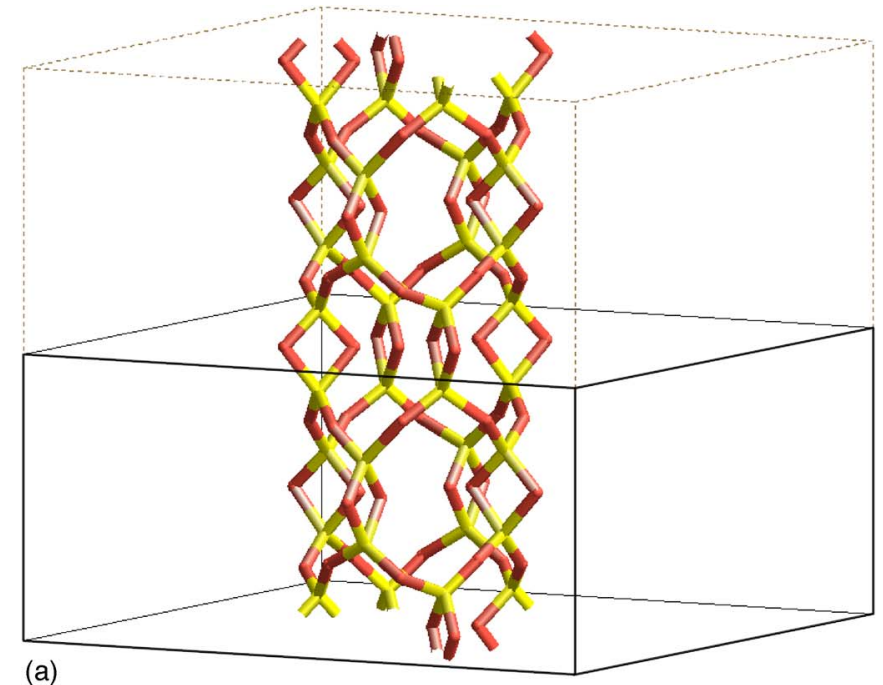

(a)

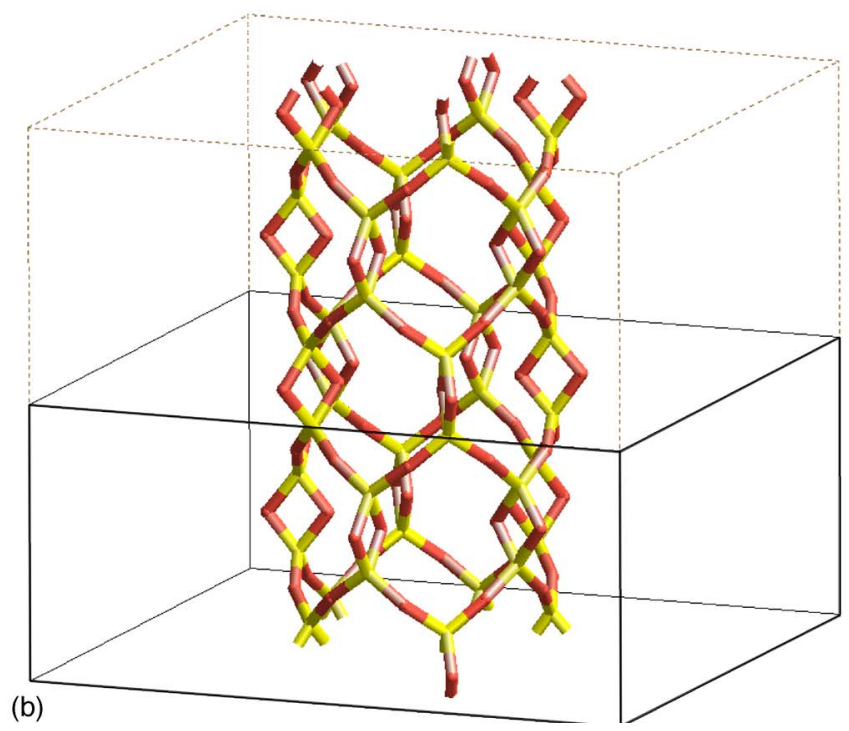

FIG. 7. (Color online) Isolated silica-based nanotubes formed from rolling up the 6-2-sheet. A $(4,0)$ tube is shown in (a), and a $(5,0)$ tube in $(b)$.

note that our lattice parameters (see Table III) also slightly differ from those reported by Hamann, ${ }^{18}$ which may further indicate that our simultaneous optimization of atomic positions and unit cell parameters may have yielded a lower energy structure than the fit of the energies of nine atomically relaxed structures with fixed unit cells to a polynomial as done in Ref. 18.

Additionally, to see the effect of local embedding strain on calculated three-ring strain in a periodic MS system we also calculate the energy of OSO: ${ }^{44}$ an all-three-ring framework material. As with the silica- $w$ MS-system for two-rings, in OSO all three-rings share all their silicon vertices directly with adjacent three-rings [see Fig. 10(b)]. This feature, as with silica- $w$, allows us to use energy units that exactly partition the material into only three-ring contributions and does not include extra embedding $\mathrm{Si}-\mathrm{O}$ bonds. In this way, using $\mathrm{eV} / \mathrm{Si}_{3 / 2} \mathrm{O}_{3}$, the strain energy per three-ring in OSO with respect to alpha quartz is calculated to be $0.24 \mathrm{eV}$, slightly

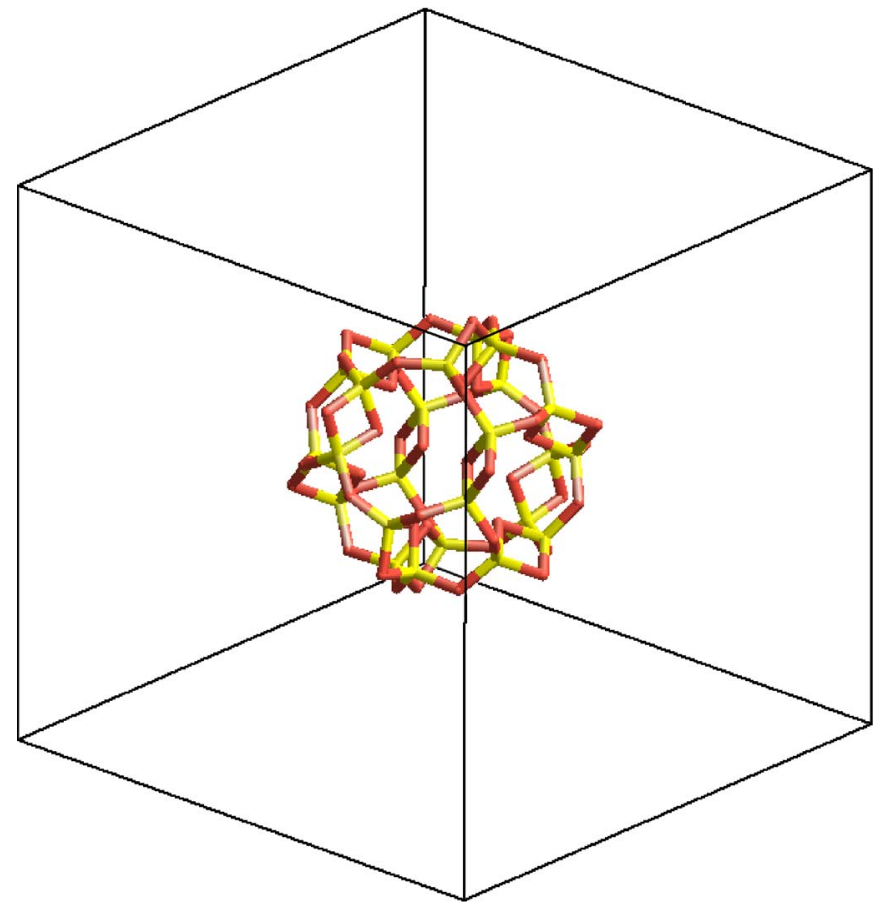

FIG. 8. (Color online) An isolated $\mathrm{Si}_{24} \mathrm{O}_{48}$ silica fullerene analog.

higher than for the L3R material. As in the case of comparing the isolated two-ring chain with the isolated 6-2-sheet, the probable reason for the small difference is the increased strain induced within the three-rings in OSO due to the relatively strained embedding environment.

\section{DISCUSSION AND CONCLUSIONS}

Using the periodic subtractive methodology, we have attempted to refine the requirements with respect to deriving

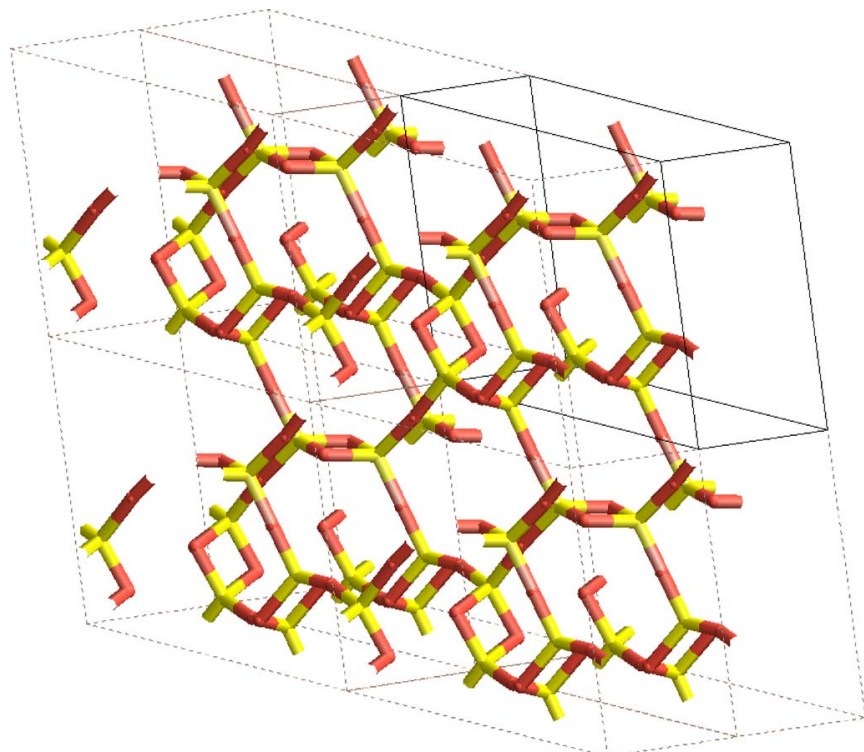

FIG. 9. (Color online) A three-dimensionally connected material containing two-rings and embedding six-rings. 

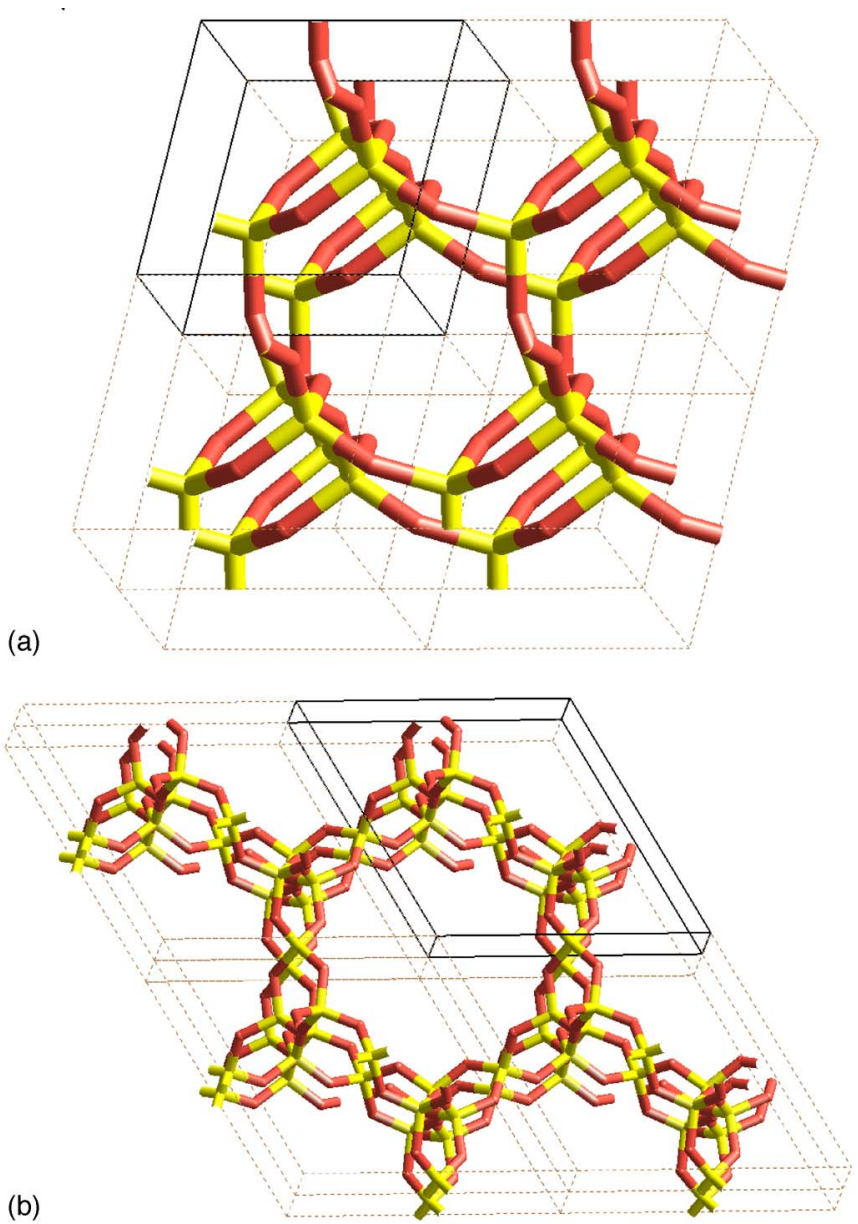

FIG. 10. (Color online) Two three-ring-containing materials: to the left is the L3R material proposed by Hamann (Ref. 18) with embedding seven-rings, and to the right the OSO (Ref. 44) framework material consisting only of connected three-rings.

the minimal intrinsic strain energies of small $(\mathrm{SiO})_{N} N<4$ rings. Through detailed considerations of two-ringcontaining materials in particular, the type of MS system and the level of theory are both found to be important. The usefulness of a MS system in periodic subtractive calculations of energies of small rings is strongly dependent on the nature of the embedding environment of the rings, which is reflected in the energy units employed. In such calculations, gradient corrected (e.g., PW91) or hybrid functionals (e.g., B3LYP) appear to perform better than local density functionals and HF, the latter both giving energies too high and poor agreement with available experimental data.

We note that our final results for two and three-ring strain (1.37 and $0.21 \mathrm{eV}$ respectively) are considerably lower than most estimates based on cluster subtractive calculations [e.g., 1.83 (Ref. 15)-1.85 eV (Ref. 16) for two-rings and 0.27 (Ref. 16)-0.81 eV (Ref. 15) for three-rings]. In these calculations, however, HF was employed, which is known from our calculations on two-ring chains above to give relatively high estimates of strain energy. In contrast, the study in Ref. 17, using the B3LYP functional and DFT cluster subtractive calculations, gives a relatively low two-ring strain energy of $1.42 \mathrm{eV}$. In these calculations it is assumed that a symmetric planar $\mathrm{OH}$-terminated four-ring $\left(\mathrm{Si}_{4} \mathrm{O}_{4}\right)$ is a suitable $\mathrm{U}$ system. In the calculations of Uchino et al. the strain in a puckered four-ring is calculated to indeed be very low $(0.02 \mathrm{eV})$; however, in a more planar conformation, the estimated inherent strain energy is thought to be considerably higher $\left(\sim 0.16 \mathrm{eV} .{ }^{45}\right)$. Calculating the energy of the planar ring and a relaxed puckered $\mathrm{OH}$-terminated four-ring cluster at exactly the same level of theory reported in Ref. 17 verifies that the planar ring is an energetically higher-lying minima. We also note that the reported O-Si-O angles of $138^{\circ}$ in Ref. 17 seem unphysically high and so possibly erroneous. Our calculations of the same planar four-ring system using exactly the same level of theory give an O-Si-O angle of $112.7^{\circ}$, giving a sum of internal angles (together with the reproduced $\mathrm{Si}-\mathrm{O}-\mathrm{Si}$ angles of $157.3^{\circ}$ ) of $1080^{\circ}$, as required for a planar system, rather than $1180^{\circ}$ as in Ref. 17 When modeling a puckered $\mathrm{OH}$-terminated four-ring cluster, one has to be careful to ensure that no significant intrahydrogen bonding occurs which would spuriously lower the energy of the system. In our puckered four-ring the distances between all possible $\mathrm{O} \cdots \mathrm{H}$-interacting pairs on $\mathrm{OH}$ groups on neighboring silicon atoms are all greater than $3.85 \AA$, ensuring a minimal hydrogen bonding contribution to the energy. Using the puckered four-ring minima as a slightly improved U system, and an OH-terminated two-ring MS system calculated at the same level of theory, the calculated two-ring strain energy increases by $\sim 6 \%$ (1.51 eV for a two-ring) with respect to the reported result using a planar four-ring $\mathrm{U}$ system. ${ }^{17}$ This small correction to the original result gives quite a high estimate of two-ring strain with respect to our periodic calcu-

TABLE III. Summary of periodic DFT calculations of different structures with high concentrations of two-rings. Our calculations used the VASP code with the PW91 functional and employed a $3 \times 3 \times 3$ Monkhorst-Pack grid of $k$ points. The calculation of Ref. 18 used a custom plane-wave DF code (Ref. 38) with the PW91 functional. For the OSO MS systems the min/max range of three-ring geometric parameters is given.

\begin{tabular}{|c|c|c|c|c|c|c|c|c|c|c|}
\hline MS system & $a$ & $b$ & $c$ & $\alpha$ & $\beta$ & $\gamma$ & $\begin{array}{l}\mathrm{Si}-\mathrm{O} \text { bond } \\
\text { length }(\AA)\end{array}$ & $\begin{array}{l}\mathrm{O}-\mathrm{Si}-\mathrm{O} \\
\text { angle }\left({ }^{\circ}\right)\end{array}$ & $\begin{array}{l}\text { Si-O-Si } \\
\text { angle }\left({ }^{\circ}\right)\end{array}$ & $\begin{array}{l}\text { Energy per } \\
\text { three-ring } \\
(\mathrm{eV})\end{array}$ \\
\hline L3R material & 5.33 & 5.33 & 5.33 & 97.6 & 97.6 & 97.6 & 1.637 & 107.9 & 132.1 & 0.21 \\
\hline L3R material (Ref. 18) & 5.31 & 5.31 & 5.31 & 96.9 & 96.9 & 96.9 & 1.640 & 109.0 & 131.0 & 0.25 \\
\hline \multirow[t]{2}{*}{ OSO framework } & & & & & & & $1.635-$ & $107.3-$ & $129.4-$ & \\
\hline & 10.31 & 10.31 & 7.78 & 90.0 & 90.0 & 120.0 & 1.638 & 107.5 & 129.7 & 0.24 \\
\hline
\end{tabular}


lations, but which is still lower than all HF estimates indicating, as we have also found above, the relative superiority of B3LYP in small-ring calculations. We have also repeated the calculation, using the same methodology as in Ref. 17 with respect to three-rings, with the puckered four-ring $U$ system. In our three-ring we observe a very small puckering of the three-ring, giving $\mathrm{Si}-\mathrm{O}-\mathrm{Si}$ angles of $130^{\circ}$ rather than $131^{\circ}$ in the planar three-ring in Ref. 17 with otherwise apparently identical geometries. Using our puckered four-ring as a MS system yields a three-ring strain of $0.22 \mathrm{eV}$, in excellent agreement with our periodic calculations $(0.21 \mathrm{eV})$ and significantly lower than the lowest values predicted from HF-cluster calculations $\left(0.27 \mathrm{eV}^{16}\right)$. At once this confirms both our low three-ring strain energy prediction using periodic PW91 calculations and the improvement of using hybrid and/or gradient corrected functionals over HF. The agreement between our cluster and periodic DF calculations of three-ring strain energy contrasts with the corresponding predictions for two-ring energies, indicating the importance of incorporating a realistic environment for assessments of strain in the latter. All our cluster calculations were performed with no symmetry constraints using the GAMESS-UK code. ${ }^{46}$

Although we advocate the use of gradient corrected and/or hybrid density functionals for more accurate small ring calculations, the DF approach cannot, with current functionals, incorporate all electronic degrees of freedom that could be of significance in small-ring systems. In an effort to go beyond the DF approximation while incorporating a realistic local embedding, Mukhopadhyay et al. ${ }^{24}$ used an $a b$ initio incremental cluster technique to estimate accurate strain energies of two-rings in isolated two-ring chains with respect to alpha quartz. For the $\mathrm{MS}$ system an $\mathrm{OH}$ terminated $\mathrm{Si}_{8} \mathrm{O}_{16}$ chain cluster, energy minimized at a coupled cluster (CCSD) level, was employed. For the U system a $\mathrm{H}-$ and $\mathrm{OH}-$ terminated fragment of alpha quartz was employed, fixed at the experimentaly determined geometry. Although one may argue if a terminated two-ring chain is truly representative of an infinite two-ring chain, the latter allowing for greater geometric relaxation and possessing long-range electrostatic interactions not present in the former, it is clear that such a relaxed linear cluster will possess two-rings in a relatively low-energy state. In the employed MS system, however, no geometric relaxation is performed and thus one must rely on the fixed structure of the terminated cluster to accurately represent the energetics of the extended alpha-quartz system. In this latter case the longrange Madelung field is not taken into account, which, in the real material, would contribute to stabilizing the electronic state in the fixed bonding configuration chosen, but, probably more significantly, the terminated cluster will inevitably possess an energy penalizing dipole. In the real material, under normal conditions, the dipole is excluded and does not contribute to raising the energy of the material. These two factors would likely result in a significant increase in the energy of the fixed cluster $\mathrm{U}$ system employed and thus in a reduced energy difference between the MS and U systems in the subtractive calculation of two-ring strain. The reported value of two-ring strain in Ref. 24 is, in fact, by far the lowest ever reported $\left(0.61 \mathrm{eV} / \mathrm{SiO}_{2}\right)$, being over half that of our periodic
DF calculations on isolated two-ring chains (see Table I) and is, we feel, at least partially due to the above reasons, probably inaccurate. One other possible reason for such a low reported value could simply be due to an error in the reported units, which as we showed above, should be chosen with care for two-ring chains. This, however, seems unlikely as in the same study the strain energy of a two-ring in an isolated chain, employing a periodic HF calculation, is also given as $2.12 \mathrm{eV} / \mathrm{SiO}_{2}$, which although fairly high, is of a similar order of magnitude to our, and other, HF-calculated estimates [1.73-1.85 (Ref. 16) eV/ $\left.\mathrm{SiO}_{2}\right]$.

In summary, we have carefully examined the use of various MS and U systems within the subtractive methodology of calculating small-ring strain and used the analysis to optimize the MS system in our periodic DF calculations. Through this procedure we attempt to provide refined benchmark values for two- and three-ring strain. For two-rings the 6-2-3D material isolated 6-2-sheet, and the isolated chain provide MS systems yielding a small range of two-ring strain energies $(1.33-1.37 \mathrm{eV})$. The slightly different predictions are likely to be mainly due to the variability in the strain in the immediate embedding environment in each MS system. Considering the very minor changes in two-ring geometry in each MS system (see Tables I and II), however, the longrange electrostatics of the MS system in question may also contribute to the differences, making it difficult to discern the best intrinsic two-ring strain measure. Considering that tworings in real siliceous materials always lie within an extended bonded $\mathrm{SiO}_{2}$ network, it is perhaps better to use this range of values (containing the most-strained and least-strained immediate embedding environments) as a guide to the small variability of two-ring strain energies in real materials. We further note that these optimal estimates of two-ring strain are only slightly lower than the estimate by Ceresoli $e t$ al. $(1.38 \mathrm{eV})$ (Ref. 3), mutually confirming the periodic subtractive methodology employed in each case. For three-rings we confirm the methdology of Hamann ${ }^{18}$ in the use of the L3R material as an appropriate periodic MS system for calculating 3-ring strain, obtaining a value of $0.21 \mathrm{eV}$. It is hoped that the refined small-ring strain values reported herein will be of use in experimental and theoretical studies of strain silica systems.

\section{ACKNOWLEDGMENTS}

We thank A. A. Sokol and F. Cora for useful discussions. D. R. Hamann, M. Bernasconi, and A. B. Mukhopadhyay are acknowledged for clarifying aspects of their work. Financial support from the Spanish Ministerio de Ciencia y Tecnologia [Projects NBA05-33-001 and CTQ2005-08459-CO2-01 and the Ramón y Cajal program (S.T.B. and I de P. R. M.)] and, in part, from the Generalitat de Catalunya (Project 2005SGR-00697 and Distinció per a la Promoció de la Recerca Universitària de la Generalitat de Catalunya granted to F.I.) is fully acknowledged. Part of the computer time was provided by the Centre de Supercomputació de Catalunya, CESCA, Centre Europeu de Paral.lelisme de Barcelona, CEPBA, and CEBPA-IBM-Research Institute, CIRI, through generous grants from Universitat de Barcelona, Fundació Catalana per a la Recerca, and CIRI. 
${ }^{1}$ V. V. Murashov, J. Phys. Chem. B 109, 4144 (2005).

${ }^{2}$ T. Bucko, L. Benco, and J. Hafner, J. Chem. Phys. 118, 8437 (2003).

${ }^{3}$ D. Ceresoli, M. Bernasconi, S. Iarlori, M. Parrinello, and E. Tosatti, Phys. Rev. Lett. 84, 3887 (2000).

${ }^{4}$ C. M. Chaing, B. R. Zegarksi, and L. H. Dubois, J. Phys. Chem. 97, 6948 (1993).

${ }^{5}$ A. Roder, W. Kob, and K. Binder, J. Chem. Phys. 114, 7602 (2001).

${ }^{6}$ A. M. Ferrari, E. Garrone, G. Spoto, P. Ugliengo, and A. Zecchina, Surf. Sci. 323, 151 (1995).

${ }^{7}$ A. S. D'Souza and C. G. Pantano, J. Am. Ceram. Soc. 85, 1499 (2002).

${ }^{8}$ A. E. Geisberger and F. L. Galeener, Phys. Rev. B 28, 3266 (1983).

${ }^{9}$ T. Uchino, A. Aboshi, S. Kohara, Y. Ohishi, M. Sakashita, and K. Aoki, Phys. Rev. B 69, 155409 (2004).

${ }^{10}$ T. A. Michalske and S. W. Freiman, J. Am. Ceram. Soc. 66, 284 (1983).

${ }^{11}$ T. Uchino and T. Yoko, Phys. Rev. B 68, 041201(R) (2003).

${ }^{12}$ K. Awazu and H. Kawazoe, J. Appl. Phys. 94, 6243 (2005).

${ }^{13}$ T. Kudo and S. Nagase, J. Am. Chem. Soc. 107, 2589 (1984).

${ }^{14}$ M. O'Keefe and G. V. Gibbs, J. Chem. Phys. 81, 876 (1984); 82, 2514 (1985).

${ }^{15}$ M. O'Keefe and G. V. Gibbs, J. Phys. Chem. 89, 4574 (1985).

${ }^{16}$ T. Uchino, Y. Kitagawa, and T. Yoko, Phys. Rev. B 61, 234 (2000).

${ }^{17}$ N. Lopez, M. Vitiello, F. Illas, and G. Pacchioni, J. Non-Cryst. Solids 271, 56 (2000).

${ }^{18}$ D. R. Hamann, Phys. Rev. B 55, 14784 (1997).

${ }^{19}$ A. Weiss, Z. Anorg. Allg. Chem. 276, 95 (1954).

${ }^{20} \mathrm{We}$ are grateful to Professor D. R. Hamman for confirming this small typographical error through a private communication.

${ }^{21}$ G. Kresse and J. Hafner, Phys. Rev. B 47, 558 (1993).

${ }^{22}$ V. R. Saunders, R. Dovesi, C. Roetti, R. Orlando, C. M. ZicovichWilson, N. M. Harrison, K. Doll, B. Civalleri, I. J. Bush, Ph. D'Arco, M. Llunell, CRYSTALzo03 User's Manual (University of Torino, Torino, Italy, 2003).

${ }^{23}$ L. B. Hansen et al. computer-code DACAPO package (v. 2.7), The Center for Atomic-Scale Materials Physics (CAMP), Technical University of Denmark, Lyngby, Denmark.

${ }^{24}$ A. B. Mukhopadhyay, M. Dolg, and C. Oligschleger, J. Chem. Phys. 120, 8738 (2004).
${ }^{25}$ P. E. Blöch, Phys. Rev. B 50, 17953 (1994).

${ }^{26}$ J. P. Perdew and Y. Wang, Phys. Rev. B 33, 8800 (1986).

${ }^{27}$ H. J. Monkhorst and J. D. Pack, Phys. Rev. B 13, 5188 (1967).

${ }^{28}$ D. Vanderbilt, Phys. Rev. B 41, 7892 (1990).

${ }^{29}$ C. Pisani, R. Dovesi, and C. Roetti, Hartree-Fock Ab Initio Treatment of Crystalline Systems, Vol. 48 of Lecture Notes in Chemistry (Springer-Verlag, Heidelberg, 1988).

${ }^{30}$ Quantum Mechanical Ab-Initio Calculation of the Properties of Crystalline Materials, Lecture Notes in Chemistry, Vol. 67, edited by C. Pisani (Springer-Verlag, Heidelberg, 1992).

${ }^{31}$ M. D. Towler, A. Zupan, and M. Causa, Comput. Phys. Commun. 98, 181 (1996).

${ }^{32}$ J. C. Slater, The Self-Consistent Field for Molecules and Solids; Quantum Theory of Molecules and Solids (McGraw-Hill, New York, 1974), Vol. 4.

${ }^{33}$ S. H. Vosko, L. Wilk, and M. Nusair, Can. J. Phys. 58, 1200 (1980).

${ }^{34}$ J. P. Perdew and Y Wang, Phys. Rev. B 45, 13244 (1992).

${ }^{35}$ A. D. Becke, Phys. Rev. A 38, 3098 (1988).

${ }^{36}$ C. Lee, W. Yang, and R. G. Parr, Phys. Rev. B 37, 785 (1988).

${ }^{37}$ M. Catti, B. Civalleri, and P. Ugliengo, J. Phys. Chem. B 104, 7259 (2000); B. Civalleri, C. M. Zicovich-Wilson, P. Ugliengo, V. R. Saunders, and R. Dovesi, Chem. Phys. Lett. 292, 394 (1998).

${ }^{38}$ D. R. Hamman, Phys. Rev. B 51, 7337 (1995); 51, 9508 (1995).

${ }^{39} \mathrm{X} . \mathrm{Xu}$ and W. A. Goddard III, J. Phys. Chem. A 108, 2305 (2004).

${ }^{40}$ S. T. Bromley, M. A. Zwijnenburg, and Th. Maschmeyer, Phys. Rev. Lett. 90, 035502 (2003).

${ }^{41}$ C. R. Martin, Chem. Mater. 8, 1739 (1996).

${ }^{42}$ J. M. Schulman, R. L. Disch, M. A. Miller, and R. C. Peck, Chem. Phys. Lett. 141, 45 (1987).

${ }^{43}$ S. T. Bromley, Nano Lett. 4, 1427 (2004).

${ }^{44}$ A. K. Cheetham, H. Fjellvag, T. E. Gier, K. O. Kongshaug, K. P. Lillerud, and G. D. Stucky, Stud. Surf. Sci. Catal. 135, 158 (2001).

${ }^{45}$ F. L. Galeener, J. Non-Cryst. Solids 49, 53 (1982).

${ }^{46}$ GAMESS-UK is a package of $a b$ initio programs written by M. F. Guest, J. H. van Lenthe, J. Kendrick, and P. Sherwood, with contributions from R. D. Amos, R. J. Buenker, H. van Dam, M. Dupuis, N. C. Handy, I. H. Hillier, P. J. Knowles, V. BonacicKoutecky, W. von Niessen, R. J. Harrison, A. P. Rendell, V. R. Saunders, K. Schoffel, A. J. Stone and D. Tozer. 